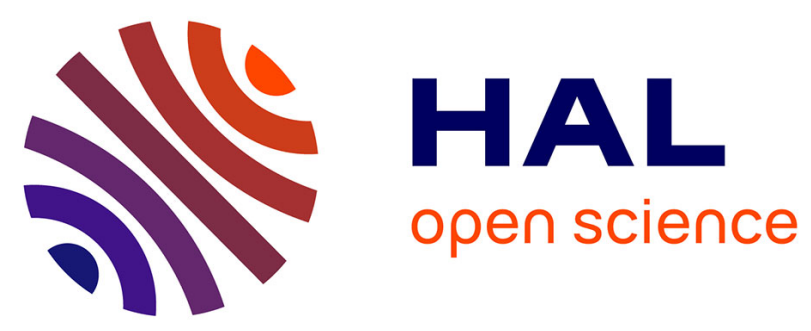

\title{
Full-potential calculations of the temperature dependence of self-diffusion coefficients : application to Cs
}

D. Fuks, J. Pelleg, S. Dorfman, S. Rashkeev

\section{- To cite this version:}

D. Fuks, J. Pelleg, S. Dorfman, S. Rashkeev. Full-potential calculations of the temperature dependence of self-diffusion coefficients: application to Cs. Journal de Physique IV Proceedings, 1993, 03 (C7), pp.C7-549-C7-552. 10.1051/jp4:1993790 . jpa-00252210

\section{HAL Id: jpa-00252210 https://hal.science/jpa-00252210}

Submitted on 1 Jan 1993

HAL is a multi-disciplinary open access archive for the deposit and dissemination of scientific research documents, whether they are published or not. The documents may come from teaching and research institutions in France or abroad, or from public or private research centers.
L'archive ouverte pluridisciplinaire HAL, est destinée au dépôt et à la diffusion de documents scientifiques de niveau recherche, publiés ou non, émanant des établissements d'enseignement et de recherche français ou étrangers, des laboratoires publics ou privés. 


\title{
Full-potential calculations of the temperature dependence of self-diffusion coefficients: application to $\mathrm{Cs}$
}

\author{
D. FUKS, J. PELLEG, S. DORFMAN* and S. RASHKEEV ${ }^{* *}$
}

Materials Engineering Department, Ben-Gurion University of the Negev, P.O. Box 653, 84105 Beer Sheva, Israel ${ }^{*}$ Department of Physics, Technion, Israel Institute of Technology, 32000 Haifa, Israel

${ }^{* *}$ Institute of Theoretical Physics, Chalmers University of Technology, 41296 Göteborg, Sweden

\begin{abstract}
The change in slope of the Arrhenius curve for the diffusion coefficient as a function of temperature is discussed and its occurance is explained by vacancies concentration dependence of the formation energy. It is shown that a sign of the mixing energy parameter of the regular solid solution of vacancies in pure metals is closely connected with the sign of the curvature of the diffusion plot. For the analysis of this plot as well as for the estimation of other parameters of self diffusion of $b c c$ Cs we used the total energy calculations based on the Full Potential LMTO method.
\end{abstract}

Microscopical calculations of the diffusion parameters are of great interest because of the obvious difficulties of their evaluation from experiments, especially at low temperatures. The processes of the formation and the migration of vacancies in the bulk can be described by the energies of formation and migration, $E_{v}$ and $E_{\mu}$, respectively. These values determine a temperature behaviour of the self-diffusion coefficient [1].

In the case of Shottky-type vacancies the sum $\left(E_{v}+E_{\mu}\right)$ is the activation energy, $E_{\text {act }}$, for the self-diffusion. $E_{v}$ and $E_{\mu}$ are usualy considered as temperature independent constants but such a dependence could be a reason of a deviation of the curve $\ln D=f\left(\frac{1}{T}\right)$ from a straight line. The problem of the analysis of the curved self-diffusion plots still represent a challenge for theory.

There exist two main approaches in the interpretation of experimental data for "abnormal" metals, i.e. metals with nonlinear dependence of $\ln D$ vs $\frac{1}{T}$. One approach is based on an assumption of coexistence of more than one diffusion mechanisms, for example, of mono- and divacancy migration processes [2], where the mono- and di-vacancy contributions have been described by two activation enthalpies. In this case the self-diffusion coefficient has been written as a sum of two exponential terms with different enthalpies for these possible configurations. A quite different method of analysis of the curved Arrhenius plot in metals was considered by Gilder and Lazarus[3]. Their idea was that this curvature is connected with the dependence of the activation volume on temperature $\left(\Omega_{a c t} \sim T\right)$, although such a suggestion is not motivated enough and, generally speaking, is not true for metals (see, e.g., Ref.[4]). It was shown that in this model the curved self-cliffusion plot can be explained with the assumption of a single vacancy mechanism. The curved diffusivity plot can be explained with a reasonable accuracy on the basis of this approach However, it was shown that in low-temperature region the enthropy calculated from

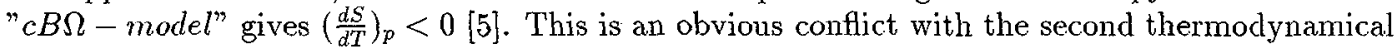
law.

In this paper we present a model with temperature dependent $E_{v}$. The ab initio estimation of $E_{v}$ for $b c c-C s$ will be carried out. In the following model the temperature dependence will be introduced by taking into account the possible dependence of $E_{v}$ on vacancies concentration. $E_{\mu}$ is considered concentration independent. This parameter is connected with the movement of the atom from the lattice site to the nearest neighbour vacancy. The vacancy concentration in metals is usually very small up to the melting temperature, and the probability of the situation 
with two or more vacancies in the neighbourhood environment of the jumping atom is small also. Therefore, the height of the potential barrier for such a jump may be considered independent on vacancies concentration.

The energy of the vacancy formation at lattice sites depends on the vacancy concentration in the bulk. This results from a decrease of average interatomic binding forces with the removing of the atoms. Temperature dependence of the vacancy concentration, $c_{v}$, is usually described in a form of the Boltzman distribution (BD), but this relation becomes more complicated if $E_{v}$ is a. function of $c_{v}$. Because of this fact the temperature dependent change in the slope of the diffusion coefficient, $D$, can be discussed without consideration of any particular diffusion mechanism. We consider only processes with formation of single vacancies (mono- vacancy mechanism), and formation processes at different lattice sites are assumed to be independent.

Assuming that vacancies in pure metals form a completely disordered regular solid solution of holes in the bulk we can write the free energy, $\mathcal{F}$, of such a solution as a sum of the energy term, $E$, and the configuration entropy of the vacancy-atom "alloy". Using the equilibrium condition $\left(d \mathcal{F} / d c_{v}=0\right)$ one immediately gets

$$
c_{v}=\left[1+\exp \left(\frac{d E}{d c_{v}} \cdot \frac{1}{k T}\right)\right]^{-1} .
$$

This expression is more general than $\mathrm{BD}$. It can be reduced to $\mathrm{BD}$ if one assumes the linear approximation for the concentration dependence of $E$ with $E_{v}$ independent on $c_{v}$.

In a general case, the dependence of $\ln D$ on $\frac{1}{T}$ is not linear, and experimental observations indicate that the change in slope may be essential. This dependence on $\frac{1}{T}$ can be obtained in a way which is more pliysically transparent than previous approaches $[2,4]$. Let us assume, that $E_{v}$ may be expressed as a polynomial function of $c_{v}$,

$$
E_{v}=E_{0}^{v}+c_{v} E_{1}^{v}+c_{v}^{2} E_{2}^{v}+\ldots
$$

Here $E_{1}^{v}$ and $E_{2}^{v}$ are expansion coefficients. Using Eq.(1) we can rewrite the diffusion coefficient as

$$
D \sim \exp \left\{-\frac{1}{k T}\left[E_{0}^{v}+E_{\mu}+\frac{E_{1}^{v}}{1+\exp \left(\frac{E_{0}^{v}}{k T}\right)}+\frac{E_{2}^{v}}{\left[1+\exp \left(\frac{E_{0}^{v}}{k T}\right)\right]^{2}}+\ldots\right]\right\} .
$$

We neglected the concentration dependent terms in the internal exponents of Eq.(3). From this expression it is possible to analyse the limiting cases at high and low temperatures. If we neglect the term with $E_{2}^{v}$ and higher terms for simplicity we obtain the different logarithmic dependence of $D$ on $\frac{1}{T}$ at different temperature limits: a) at low temperatures,

$$
\ln D \sim-\frac{E_{0}^{v}+E_{\mu}}{k T} .
$$

This is the well-known Arrhenius dependence of $\ln D$ vs $\frac{1}{T}$ at low temperatures. b)at high temperatures,

$$
\text { In } D \sim-\frac{E_{0}^{v}+E_{\mu}+\frac{E_{1}^{v}}{2}}{k T} .
$$

The magnitude of $E_{1}^{v}$ in Eq.(5) determines the change in the slope of the Arrhenius curve at high temperatures. This result was obtained without any assumption about diffusion mechanism and without traditional assumption about switching on di-vacancy formation processes with the growth of temperature. 
Now we can clear up the physical meaning of the term including $E_{1}^{v}$ using the model of regular solid solution which seems to be reasonable in a case of small concentration of vacancies. This model considers "atom-vacancy", solid solution, and the energy is expressed in the form,

$$
E\left(c_{v}\right)=c_{v} \cdot E_{v_{p}}+\left(1-c_{v}\right) \cdot E_{0}+U \cdot c_{v}\left(1-c_{v}\right) .
$$

Here $E_{v_{p}}$ is the energy of vacancy and the last term represents the mixing energy of such a solution. Comparing the $c_{v}^{2}$ terms in Eqs.(2) and (6) one gets immediately $E_{1}^{v}=-U$. If $E_{1}^{v}$ is negative $(U>0)$ we have a normal behaviour for diffusion coefficient, with positively curved Arrhenius plot. If $E_{1}^{v}$ is positive, the negatively curved Arrhenius plot will be obtained. In the theory of regular solid solutions for alloys the sign of $U$ shows the energetical preference of the disordered solution in comparison with the geterogeneous mixture of components. If $U<0$, the disordered solid solution of vacancies is energetically favorable in comparison with the noninteracting holes-atoms mixture, and the function $D=f(1 / T)$ will be negatively curved. Such a situation was indeed observed in some metals which show structural phase transitions when temperature increases, for example, in $\alpha-Z r[6]$ with hep - bcc transition at $\sim 870^{\circ} \mathrm{C}$.

For microscopical estimations of the diffusion parameters one can try to use the mean-field approximation for the "vacancy-atom" solution. In the framework of such an approach the presence of vacancies in the bulk can be simulated by calculation of the total energy, $E^{\text {tot }}\left(c_{v}\right)$, in ideal crystal which consists of "quasiatoms" with effective atomic charge $Z^{\text {eff }}$, slightly deviating from the integer charge $Z$ corresponding to the real atoms $\left(Z^{\text {eff }}=Z \cdot\left(1-c_{v}\right)\right)$. The electroneutrality conditions will be automatically satisfied in such an approach. This method gives a possibility to estimate $a b$ initio the dependence of the total energy on vacancy concentration. Assuming that $E^{t o t}\left(c_{v}\right)$ is also a polynomial function of $c_{v}$ we can prepare an expansion of the form of Eq. (2),

$$
E^{t o t}\left(c_{v}\right)=E^{t o t}(0)+c_{v} E_{0}^{t o t}+c_{v}^{2} E_{1}^{t o t}+\ldots
$$

In a general case, there is no reason to indentify the coefficients of this expansion with those given by Eq.(2) because the vacancy formation energy, $E_{v}$, depends mostly on the interaction of a given atom with its environment, i.e. the total energy $E^{\text {tot }}$ contains more information. But an assumption about the proportionality of the coefficients $E_{i}^{t o t}$ and $E_{i}^{v}(i=0,1, \ldots)$ gives a. possibility to analyze qualitatively parameters of the self-diffusion process from the total energy calculations with a reasonable accuracy.

We performed the calculations of the total energy with different vacancy concentrations in the framework of Full Potential Linear Muffin-Tin Orbital (FP-LMTO) method [7]. This method allows to feel the differences in total energies of the order of $10^{-5} R y$ for the bulk deformations of the crystal. The relativistic corrections were included using the scalar-relativistic scheme (without the spin-orbit coupling). Calculations have been performed on the mesh corresponding to about $300 \vec{k}$ :-points in the irreducible wedge of the Brillouin zone, the Gunnarsson-Lundquist exchangecorrelation potentialhas been used. The band structure is practically idendical with the results of the previous papers [8]

The total energies of the crystal were calculated for different lattice constants of bcc-Cs and different small variations of "quasiatomic" charge, $Z^{\text {eff }}$. The calculated value of equilibrium volume for $Z=55$ is $\Omega_{0}=774.3 a . u .^{3}$. This is in a good agreement with experimental data[9] $\left(\Omega_{e x p}=782.2 a \cdot u .^{3}\right)$. The total energy of $b c c$-Cs grows with growing of vacancy concentration for all the used atomic volumes.

Doing the fitting procedure of these energies to the expansion (7) one can estimate the value of the ratio $\alpha=E_{0}^{\text {tot }} / E_{1}^{\text {tot }}=\left|E_{0}^{v} / E_{1}^{v}\right|$. This parameter was found to be practically independent on the volume, $\alpha=1.12 \cdot 10^{-3}$, i.e. $\left|E_{1}^{\nu}\right|$ is three orders larger than $\left|E_{0}^{\nu}\right|$. This means that the convergence of the expansion (2) have to be checked very carefully. If we assume that the 
convergence of series is given by $\frac{\left|E_{0}^{v}\right|}{c_{v} \cdot\left|E_{1}^{v}\right|} \sim 10$, we can find the limiting concentration $c_{v}$ of vacancies at which the expansion from Eq. (2) is still converging, $c_{v} \sim 1.12 \cdot 10^{-2} a t \%$. Now, it is possible to receive the value of the formation energy, $E_{v}$, for $b c c$-Cs near the point $T \sim 350 \mathrm{~K}$. Suggesting that at this temperature the expansion given by Eq.(2) is converging, and using the obtained value of $c_{v}$ we obtain $E_{v}=0.274 \mathrm{eV}$ from BD. Specific heat measurements by adiabatic calorimetry yield a value of $0.28 \mathrm{eV}$ for the vacancy formation energy in $b c c \mathrm{Cs}[10]$. Calculations of Fumi[11] provide the formation energy of $0.26 \mathrm{eV}$ for $\mathrm{Cs}$, and pseudopotential calculations [12] give the value 0.33 $\mathrm{eV}$. This means that our crude estimation gives a reasonable result for $E_{y}$.

Our method gives us a possibility to prepare a qualitative analysis of the temperature dependence of the Arrhenius curve also. The value of $E_{1}^{v}$ in $b c c-C s$ is negative, while $E_{0}^{v}$ is positive. This gives a positively curved plot of $\ln D$ vs $\frac{1}{T}$. Such a behavior of the Arrhenius curve is usually observed in metals. Nevertheless, in some cases the parameter $E_{1}^{v}$ can be positive, and this can give a negative curvature of the self-diffusion plots [13]. Our method allows us to understand this feature also without any additional assumption about the diffusion mechanism.

This research was partly supported by the Israel Ministry of Science and Technology under Grant No.3616-2-92 and partly by the special program of the Israel Ministry of Absorbtion. We are greately acknowledged to S.Yu.Savrasov for his enormous support and discussions during the FP-LMTO calculations. We would like to thank G.Wendin, P.Wyder, and Ts.Maniv for their constant interest in our activity. We are grateful to M.Gillan for valuable comments.

\section{References}

[1] J.S.Kirkaldy and D.J.Young, Diffusion in the Condensed State, ( The Institute of Metals, London, 1987), $527 \mathrm{p}$.

[2] H.Mehrer, J.Nucl.Mat.Sci. 69-70, 38 (1978).

[3] H.M.Gilder and D.Lazarus, Phys.Rev. B11, 4916 (1975).

[4] P.A.Varotsos and K.D.Alexopoulos, Thermodynamics of Point Defects and Their Relation with Bulk Properties, (Amsterdam, North-Holland, 1986).

[5] M.L.Wasz and R.B.McLellan, J.Phys.Chem.Solids 53, 629 (1992)

[6] J.Horvâth, F.Dyment and H.Mehrer, J.Nucl.Mater, 126, 206 (1984).

[7] S.Yu.Savrasov and D.Yu.Savrasov, Phys. Rev. B46, 12181 (1992).

[8] M.Sigalas, et al., Phys. Rev. B 42, 11637 (1990).

[9] I.Makarenko, I.Ivanov and S.Stishov, JETP Lett., 18, 320 (1973).

[10] Ya.A.Kraftmakher and P.G.Strelkov, in Vacancies and Interstitials in Metals, edited by A.Seeger, W.Schilling and J.Diehl (North-Holland, Amsterdam, 1968) 63.

[11] F.G.Fumi, Philos. Mag. 46, 1007 (1955).

[12] P.S.Ho, Phys. Rev. B 3, 4035 (1971).

[13] W.Frank, Philos.Mag. A, 63, 897 (1991). 